\title{
Magnetic resonance cholangiopancreatography with compressed sensing at 1.5 T: clinical application for the evaluation of branch duct IPMN of the pancreas
}

\author{
Benjamin Henninger ${ }^{1}$ (D) Michael Steurer ${ }^{1} \cdot$ Michaela Plaikner $^{1} \cdot$ Elisabeth Weiland $^{2} \cdot$ Werner Jaschke $^{1} \cdot$ \\ Christian Kremser ${ }^{1}$
}

Received: 31 March 2020 / Revised: 23 April 2020 / Accepted: 29 May 2020 / Published online: 18 June 2020

(C) The Author(s) 2020

\begin{abstract}
Objectives To evaluate magnetic resonance cholangiopancreatography (MRCP) with compressed sensing (CS) for the assessment of branch duct intraductal papillary mucinous neoplasm (BD-IPMN) of the pancreas. For this purpose, conventional navigator-triggered (NT) sampling perfection with application-optimized contrast using different flip angle evolutions (SPACE) MRCP was compared with various CS-SPACE-MRCP sequences in a clinical setting.

Methods A total of 41 patients (14 male, 27 female, mean age 68 years) underwent 1.5-T MRCP for the evaluation of BD-IPMN. The MRCP protocol consisted of the following sequences: conventional NT-SPACE-MRCP, CS-SPACE-MRCP with long (BHL, $17 \mathrm{~s}$ ) and short single breath-hold (BHS, $8 \mathrm{~s}$ ), and NT-CS-SPACE-MRCP. Two board-certified radiologists evaluated image quality, duct sharpness, duct visualization, lesion conspicuity, confidence, and communication with the main pancreatic duct in consensus using a 5-point scale (1-5), with higher scores indicating better quality/delineation/confidence. Maximum intensity projection reconstructions and originally acquired data were used for evaluation. Wilcoxon signed-rank test was used to compare the intra-individual difference between sequences.

Results BHS-CS-SPACE-MRCP had the highest scores for image quality $(3.85 \pm 0.79)$, duct sharpness $(3.81 \pm 1.05)$, and duct visualization $(3.81 \pm 1.01)$. There was a significant difference compared with NT-CS-SPACE-MRCP $(p<0.05)$ but no significant difference to the standard NT-SPACE-MRCP $(p>0.05)$. Concerning diagnostic quality, BHS-CS-SPACE-MRCP had the highest scores in lesion conspicuity (3.95 \pm 0.92$)$, confidence (4.12 \pm 1.08$)$, and communication $(3.8 \pm 1.06)$, significantly higher compared with NT-SPACE-MRCP, BHL-SPACE-MRCP, and NT-CS-SPACE-MRCP $(p=<0.05)$.

Conclusions MRCP with CS 3D SPACE for the evaluation of BD-IPMN at 1.5 T provides the best results using a short breathhold sequence. This approach is feasible and an excellent alternative to standard NT 3D MRCP sequences.

Key Points

- 1.5-T MRCP with compressed sensing for the evaluation of branch duct IPMN is a feasible method.

- Short breath-hold sequences provide the best results for this purpose.
\end{abstract}

Keywords Magnetic resonance imaging · Pancreas $\cdot$ Pancreatic intraductal neoplasms

Benjamin Henninger

benjamin.henninger@i-med.ac.at

1 Department of Radiology, Medical University of Innsbruck, Anichstraße 35, 6020 Innsbruck, Austria

2 Siemens Healthcare GmbH, Henkestraße 127, 91052 Erlangen, Germany

$\begin{array}{ll}\text { Abbreviations } \\ \text { CS } & \text { Compressed sensing } \\ \text { IPMN } & \text { Intraductal papillary mucinous neoplasm } \\ \text { MIP } & \text { Maximum intensity projection } \\ \text { MRCP } & \text { Magnetic resonance cholangiopancreatography } \\ \text { MRI } & \text { Magnetic resonance imaging } \\ \text { NT } & \text { Navigator-triggered } \\ \text { SPACE } & \text { Sampling perfection with } \\ & \text { application-optimized contrast } \\ & \text { using different flip angle evolutions }\end{array}$




\section{Introduction}

Magnetic resonance imaging (MRI) is the method of choice for the evaluation of cystic pancreatic lesions [1]. Among all cystic lesions, the intraductal papillary mucinous neoplasm (IPMN) of the pancreas is increasingly the focus of attention as many of these cystic lesions are discovered incidentally [2]. MRI is mainly used for its noninvasive diagnosis, especially for the branch duct-type IPMN for detecting communication with the main pancreatic duct. Magnetic resonance cholangiopancreatography (MRCP), based on heavily T2-weighted sequences, is therefore the cornerstone and an established method [3-5]. Normally, thin-slice, isotropic, navigator-triggered (NT) 3D MRCP sequences are performed to prove ductal communication. Single-shot breath-hold 2D sequences are also frequently used, but their benefit of short acquisition time is often limited due to thicker sections and therefore the missing availability of crucial 3D reconstructions [6].

The management of IPMN is constantly evolving with currently two main strategies: the Fukuoka guidelines and the white paper of the ACR Incidental Findings Committee [2, 7]. With possible follow-up intervals of 6 months over a period of 2 years, it is important that the MRI examination is optimized for these patients. Standard and widely established MRCP protocols of the pancreas normally have long acquisition times; especially, the NT 3D MRCP sequences can take up to $7 \mathrm{~min}$, strongly depending on the cooperation (regular respiratory rhythm) of the patient [8]. Furthermore, such sequences may also result in suboptimal image quality in patients who do not have a consistent breathing pattern.

Compressed sensing (CS) is a relatively recent approach for the acceleration of image acquisition and is used in different anatomic regions [9-11]. It uses a sparse, incoherent undersampling of $\mathrm{k}$-space and a nonlinear iterative reconstruction to correct for subsampling artifacts. This can preserve image quality and enables the acquisition of fast 3D MRCP datasets, even in a single breath-hold $[12,13]$. Although the benefits of CS are obvious, it is important to evaluate such accelerated sequences for daily clinical routine with emphasize on robustness and diagnostic accuracy. In current literature, the use of CS for MRCP has already frequently been reported, mainly at $3 \mathrm{~T}[12-14]$. Nearly all studies found the CS approach feasible with comparable results to standard MRCP sequences, which have significantly longer examination time.

Our purpose was to evaluate MRCP with CS for the assessment of IPMN of the pancreas at 1.5 T. For this purpose, conventional navigator-triggered (NT) sampling perfection with application-optimized contrast using different flip angle evolutions (SPACE) 3D MRCP was compared with various prototype 3D CS-SPACE-MRCP sequences in a clinical setting.

\section{Materials and methods}

Written informed consent on the MRI was obtained from each patient. Institutional review board approval was granted by means of a general waiver for studies with retrospective data analysis (local research ethics committee, Medical University of Innsbruck; 20 February 2009). The authors who are employees of Siemens Healthcare had no control of any data and were not involved in the execution of the study.

\section{Patients}

Patients were included into our study using the following inclusion criteria: (1) patients referred to the Department of Radiology (Medical University of Innsbruck) for the MRI evaluation of a cystic lesion of the pancreas, (2) age $>18$ years, (3) no history of pancreatic surgery, (4) acquisition of our whole MRCP protocol as mentioned below, (5) suspicion of branch duct IPMN (BD-IPMN) in our current MRI report (positive communication of the cystic lesion with the main pancreatic duct in at least one sequence, no previous MRI available) or in other imaging procedures (e.g., computed tomography (CT), endoscopic ultrasound (EUS), or previous MRI), i.e., positive communication of the cystic lesion with the main pancreatic duct, mentioned in the report (see Fig. 1).

After searching our internal database, we retrospectively included 41 patients ( 27 female; 14 male; median age, 69 years; range, 46-89 years) between March 2018 and December 2018. Thereby, 18/41 were referred with suspicion and 23/41 for follow-up of BD-IPMN (=patients with previous MRI).

\section{MR imaging}

All studies were performed on a 1.5-T MR system (MAGNETOM Avanto ${ }^{\text {fit }}$, Siemens Healthcare) using an 18element body matrix coil and a 32-element spine coil. Patients were scanned in supine position. Standard examination preparation for each patient included oral administration of 200 $\mathrm{mL}$ of water with one tablet of Supradyn forte (Bayer) $10 \mathrm{~min}$ prior to the examination, serving as a negative contrast agent. The MRCP protocol consisted of the following sequences in coronal orientation: (1) conventional navigatortriggered (NT) sampling perfection with applicationoptimized contrast using different flip angle evolutions (SPACE) 3D MRCP (NT-SPACE-MRCP); (2) prototype compressed sensing SPACE-MRCP with a long single breath-hold of $17 \mathrm{~s}$ (BHL-CS-SPACE-MRCP); (3) prototype compressed sensing SPACE-MRCP with a short single breath-hold of $8 \mathrm{~s}$ (BHS-CS-SPACE-MRCP); (4) prototype navigator-triggered, compressed sensing SPACE-MRCP (NT-CS-SPACE-MRCP). Details of these four sequences are provided in Table 1. Maximum intensity projection 


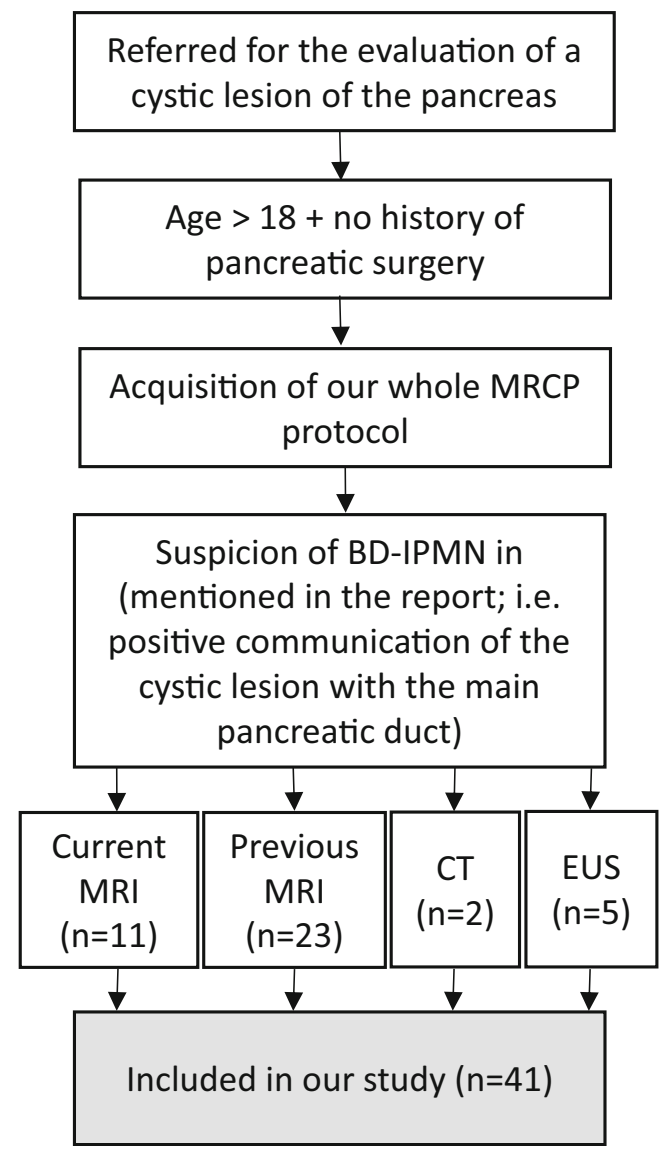

Fig. 1 Overview of patient inclusion criteria, visualized as a flow chart

(MIP) was performed for all four sequences on the satellite console of the MR unit.

In addition, our standard sequences for imaging the pancreas, including T1- and T2-weighted sequences, diffusionweighted imaging, and contrast-enhanced dynamic scans, were performed for all patients. These sequences were not utilized for the present data analysis.

For CS-MRCP, a prototype 3D SPACE sequence was used with incoherent undersampling and CS reconstruction. Incoherent undersampling was obtained using a PoissonDisk pattern [12]. For BHL-CS-SPACE-MRCP and BHSCS-SPACE-MRCP, $3.8 \% \mathrm{k}$-space data sampling was chosen leading to an acceleration factor of 26. For NT-CS-SPACEMRCP, the acceleration factor was 19 (5.4\% k-space data sampling). CS reconstruction was automatically performed in-line after image acquisition using a fast iterative softthresholding algorithm (20 iterations) together with regularization. Parameters for CS reconstruction were identical for all CS sequences.

\section{Image analysis}

All MRI scans were assessed independently by 2 radiologists with 12 years (B.H.) and 8 years (M.P.) of experience in reading MRI images of the abdomen.

Image analysis was performed in four separate reading sessions with 4-week interval to reduce potential recall bias. The four available MRCP sequences of each anonymized patient were randomized; one sequence per patient was then evaluated in one session. No data from the other performed sequences were made available during the image analysis. Readers graded all four sequences separately on a 5-point Likert-type scale in the following categories: image quality, duct sharpness, duct visualization, lesion conspicuity, confidence, and communication. In general, for the diagnostic approach (lesion conspicuity, confidence, and communication), the presence of a cystic lesion in communication with the main pancreatic duct was assessed, with higher scores indicating better results (Table 2). In case of multiple cystic lesion (> 1), only the

Table 1 Sequence parameters (all sequences were acquired in coronal orientation)

\begin{tabular}{|c|c|c|c|c|}
\hline & NT-SPACE-MRCP & BHL-CS-SPACE-MRCP & BHS-CS-SPACE-MRCP & NT-CS-SPACE-MRCP \\
\hline $\mathrm{TR}(\mathrm{ms})$ & $\begin{array}{l}\text { Variable depending on } \\
\text { the respiratory rate }\end{array}$ & 2000 & 1700 & $\begin{array}{l}\text { Variable depending on } \\
\text { the respiratory rate }\end{array}$ \\
\hline $\mathrm{TE}(\mathrm{ms})$ & 698 & 551 & 433 & 696 \\
\hline Echo train length & 270 & 162 & 230 & 180 \\
\hline Flip angle $\left(^{\circ}\right)$ & 140 & 140 & 140 & 140 \\
\hline Acquisition matrix & $353 \times 384$ & $134 \times 320$ & $115 \times 320$ & $128 \times 320$ \\
\hline Phase encoding direction & $\mathrm{R} \gg \mathrm{L}$ & $\mathrm{R} \gg \mathrm{L}$ & $\mathrm{R} \gg \mathrm{L}$ & $\mathrm{R} \gg \mathrm{L}$ \\
\hline Acceleration factor & GRAPPA 2 & CS 26 & CS 26 & CS 19 \\
\hline CS k-space data sampling (\%) & - & 3.8 & 3.8 & 5.4 \\
\hline Slice thickness (mm) & 1 & 1.2 & 1.2 & 1 \\
\hline $\mathrm{FOV}\left(\mathrm{mm}^{2}\right)$ & $320 \times 320$ & $210 \times 400$ & $210 \times 400$ & $200 \times 400$ \\
\hline Acquisition time (min) & $\begin{array}{l}\text { Variable: mean } 05: 20 ; \\
\quad \text { max. } 08: 17 ; \text { min. } 02: 53\end{array}$ & 00:17 (breath-hold) & 00:08 (breath-hold) & $\begin{array}{l}\text { Variable: mean 02:13; } \\
\text { max. 04:06; min. 01:32 }\end{array}$ \\
\hline
\end{tabular}


Table 2 Parameter scores of image analysis

\begin{tabular}{lllllll}
\hline Score & Image quality & Duct sharpness & Duct visualization & Lesion conspicuity & Confidence & Communication \\
\hline 1 & Non-diagnostic & Non-diagnostic & No visualization & Unreadable & Definitely absent & Unreadable images \\
2 & Poor & Substantial blur & $\begin{array}{c}\text { Poorly visualized, } \\
\text { limited diagnostic value }\end{array}$ & Poor & Probably absent & Indeterminate \\
3 & Fair/acceptable & $\begin{array}{c}\text { Mild blur with mild } \\
\text { image quality degradation }\end{array}$ & $\begin{array}{c}\text { Partial or blurry, } \\
\text { decreased image quality }\end{array}$ & Acceptable & Indeterminate & Poor depiction \\
4 & Good & No or minimal blur & Well visualized & Good & Probably present & Good depiction \\
5 & Excellent & No blur & Excellently visualized & Excellent & Definitely present Excellent depiction \\
\hline
\end{tabular}

largest lesion was evaluated. MIPs and the source images were evaluated together.

Finally, a consensus between both radiologists was built in a second session.

\section{Statistical analysis}

Descriptive statistics were used including mean or median, standard deviation, and range to present patient data, acquisition time, and scoring results. All statistical calculations were performed using R Project for Statistical Computing [15]. Comparison of acquisition time and scoring between different sequences was performed using a Wilcoxon signed-rank test. To assess inter-rater agreement, the Cohen's kappa coefficient (0.21-0.40, fair agreement; 0.41-0.60, moderate agreement; $0.61-0.80$, good agreement; $0.81-1.00$, perfect agreement) with equal weights was calculated using the irr-package for $\mathrm{R}$ [16]. Results were considered statistically significant for $p<0.05$.

\section{Results}

All results are summarized in Table 3.

\section{Acquisition time}

Mean acquisition time for NT-SPACE was 05:20 min (range, 02:53-08:17 $\mathrm{min}$ ) and 02:13 $\mathrm{min}$ (range, 01:32-04:06 $\mathrm{min}$ ) for the NT-CS-SPACE. The difference in acquisition time between both sequences was highly significant $(p<0.001)$.
The CS BH sequences had fixed acquisition times (one breath-hold) of $17 \mathrm{~s}$ and $8 \mathrm{~s}$ (BHL-CS-SPACE and BHSCS-SPACE).

\section{Image quality}

Image quality scores for BHS-CS-SPACE $(3.85 \pm 0.79)$ were higher than for all other evaluated sequences, with a significant difference only to NT-CS-SPACE $(3.22 \pm 0.85 ; p<0.05)$ and BHL-CS-SPACE $(3.56 \pm 0.87 ; p<0.05)$. NT-SPACE $(3.56 \pm 0.95)$ revealed a significantly higher score $(p<0.05)$ than NT-CS-SPACE $(3.22 \pm 0.85)$.

For duct sharpness and duct visualization, BHS-CSSPACE reached the highest score $(3.81 \pm 1.05$ and $3.81 \pm$ 1.01 , respectively) with a significant difference (for both) only to NT-CS-SPACE $(p<0.05)$.

For image quality, duct sharpness, and duct visualization, the difference between the NT-SPACE (our current standard sequence) and the BHS-CS-SPACE (with the highest scores) was not significant $(p>0.05)$. The NT-CS-SPACE sequence had the lowest scores among all evaluated sequences.

\section{Diagnostic quality}

The BHS-CS-SPACE sequence had the highest scores in lesion conspicuity (3.95 \pm 0.92$)$, confidence (4.12 \pm 1.08$)$, and communication $(3.98 \pm 1.06)$. All scores were significantly higher compared with NT-SPACE, BHL-CS-SPACE, and NT-CS-SPACE $(p<0.05)$.

Overall, NT-CS-SPACE reached the lowest scores concerning diagnostic quality; compared with the standard

Table 3 Results (mean score \pm standard deviation)

\begin{tabular}{|c|c|c|c|c|c|c|}
\hline Sequence & Image quality & Duct sharpness & Duct visualization & Lesion conspicuity & Confidence & Communication \\
\hline NT-SPACE & $3.56 \pm 0.95$ & $3.46 \pm 1.05$ & $3.49 \pm 1.05$ & $3.56 \pm 0.90$ & $3.71 \pm 0.93$ & $3.44 \pm 0.90$ \\
\hline BHL-CS-SPACE & $3.56 \pm 0.87$ & $3.56 \pm 1.03$ & $3.49 \pm 1.03$ & $3.59 \pm 1.07$ & $3.66 \pm 1.11$ & $3.51 \pm 1.12$ \\
\hline BHS-CS-SPACE & $3.85 \pm 0.79$ & $3.81 \pm 1.05$ & $3.81 \pm 1.01$ & $3.95 \pm 0.92$ & $4.12 \pm 1.08$ & $3.98 \pm 1.06$ \\
\hline NT-CS-SPACE & $3.22 \pm 0.85$ & $3.15 \pm 1.09$ & $3.10 \pm 0.94$ & $3.29 \pm 0.98$ & $3.32 \pm 1.13$ & $3.17 \pm 1.09$ \\
\hline
\end{tabular}


NT-SPACE, statistical significance was not reached in lesion conspicuity, confidence, and communication $(p>0.05)$.

Examples are provided in Figs. 2 and 3.

\section{Inter-rater agreement}

Inter-rater agreement was perfect for image quality, duct sharpness, duct visualization, and lesion conspicuity (weighted $k=0.826-0.915, p=<0.05$ ) and good to perfect for confidence and communication (weighted $k=0.715-0.862, p=<$ 0.05 ) (see Table 4 for detailed information).

\section{Discussion}

In our study, the BHS-SPACE-CS-MRCP sequence showed the highest scores concerning image and diagnostic quality for the evaluation of BD-IPMN. Interestingly, results from BHSSPACE-CS were noticeably better compared with the NTSPACE-CS with significantly better results concerning all evaluated categories. The main advantage is the short acquisition time with just one very short breath-hold which also seems to influence the image quality and diagnostic confidence positively for CS-MRCP sequences at $1.5 \mathrm{~T}$.

The diagnosis of the BD-IPMN is based on the proof of a communication with the main pancreatic duct. In general, this preferably is performed with thin-slice 3D sequences with longer acquisition time compared with 2D sequences [17]. We are aware that the communication with the main pancreatic duct is not always detectable with MRI in general.
However, it was not the aim of our study to evaluate the diagnostic accuracy of MRCP for the diagnosis of BDIPMN but to find the best possible and optimized MRI sequence for this purpose. In this respect, a BH-SPACE-CS sequence with a very short breath-hold seems to be an ideal sequence for the evaluation of BD-IPMN. Especially if follow-up is needed, this further reduces the required scanning time without loss of diagnostic value.

Chandarana et al compared a breath-hold accelerated SPACE sequence with sparsity-based iterative reconstruction against a conventional respiratory-triggered (RT)-SPACEMRCP at a 3-T system [12]. The overall image quality scores for BH SPARSE-SPACE were higher than those for RTSPACE, and it was concluded that a $\mathrm{BH}$ sequence showed similar or superior image quality for the pancreatic duct, despite 17-fold shorter acquisition time. This study group was one of the first to evaluate CS techniques for MRCP, and their results are comparable with ours. Most of the other so far published studies in recent literature found comparable (or even superior) results between 3D MRCP sequences performed with and without CS at $3 \mathrm{~T}$; in all cases, a shortening of the examination time with constant to better image quality was reported [13, 14, 18].

Furlan et al also assessed 3D CS-MRCP for the evaluation of pancreatic cystic lesions [19]. Their study, however, had no emphasis on IPMN and was carried out on a 3-T scanner, and the evaluated CS sequence was respiratory- or navigator-triggered. The acquisition time was decreased twofold by using CS without significantly compromising the overall image quality and the degree of artifacts.

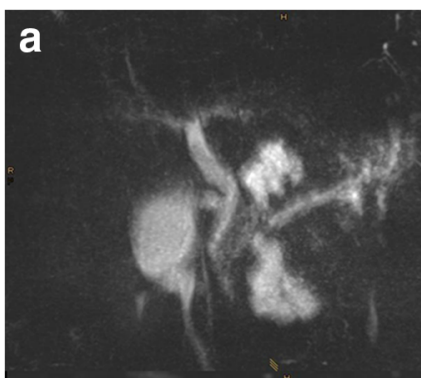

C

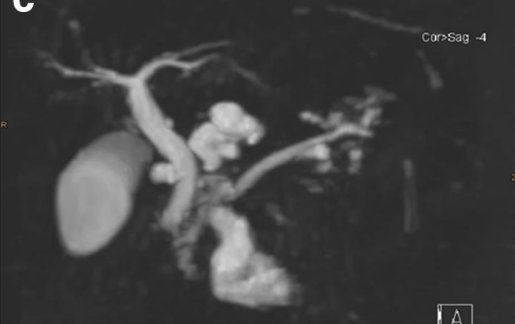

Fig. 2 A 71-year-old female patient with multiple BD-IPMN. a Conventional NT-SPACE-MRCP providing the worst quality with the longest acquisition time (04:13 min). CS-SPACE-MRCP with breathhold shows the best image quality (image $\mathbf{b}$ is BHL and image $\mathbf{c}$ is BHS). d NT-CS-SPACE-MRCP with 01:32-min acquisition time also provides only average image quality. Scores were as follows [image b

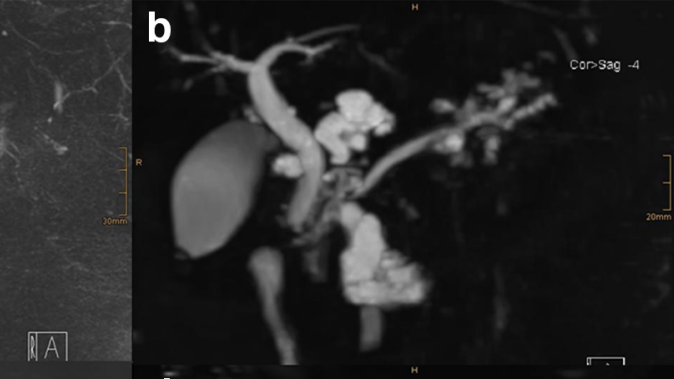

d

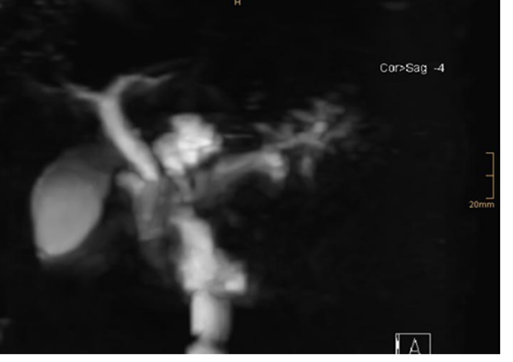

quality/duct sharpness/duct visualization/lesion conspicuity/confidence/ communication]: (a) NT-SPACE [2/2/2/2/3/2]; (b) BHL-CS-SPACE [4/4/4/4/5/4]; (c) BHS-CS-SPACE [4/5/4/4/5/5]; (d) NT-CS-SPACE [3/3/2/2/2/3] (MIPs are displayed; windowing was adjusted for optimal visualization) 
Fig. 3 A 56-year-old male patient with BD-IPMN. Difference between the sequences for source images (left) and MIPs (right) are shown. a Conventional NT-

SPACE-MRCP; the communication between the cystic lesion and the pancreatic duct cannot be visualized (circles). It can only barely be depicted with BHL-CSSPACE-MRCP (circles, b). c BHS-CS-SPACE-MRCP was able to illustrate the communication in the source images (arrow) which made the diagnosis of a BD-IPMN possible. $\mathbf{d}$ NT-CSSPACE-MRCP showed acceptable image quality, but the communication was also hard to depict due to blurring. Scores were as follows [image quality/duct sharpness/duct visualization/ lesion conspicuity/confidence/ communication]: (a) NT-SPACE [4/3/3/2/2/1]; (b) BHL-CS-

SPACE [3/1/2/2/3/2]; (c) BHSCS-SPACE [4/4/4/4/4/4]; (d) NTCS-SPACE [3/3/2/3/3/3]

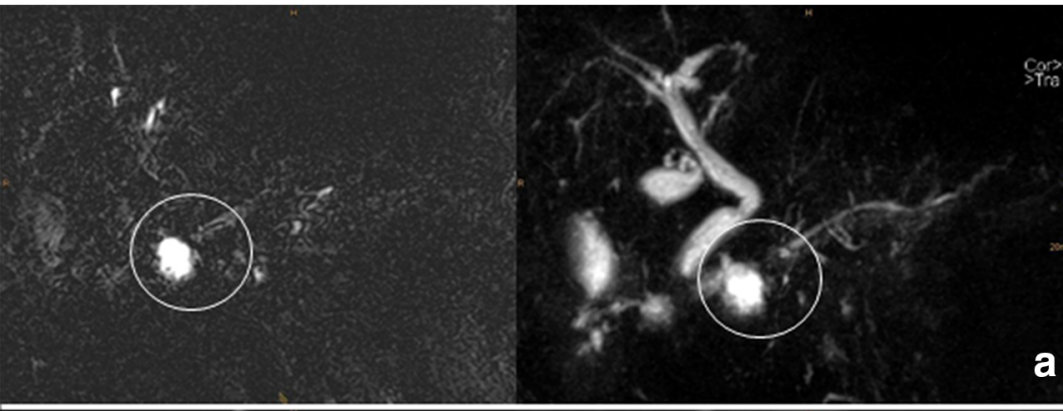

Sors:

a

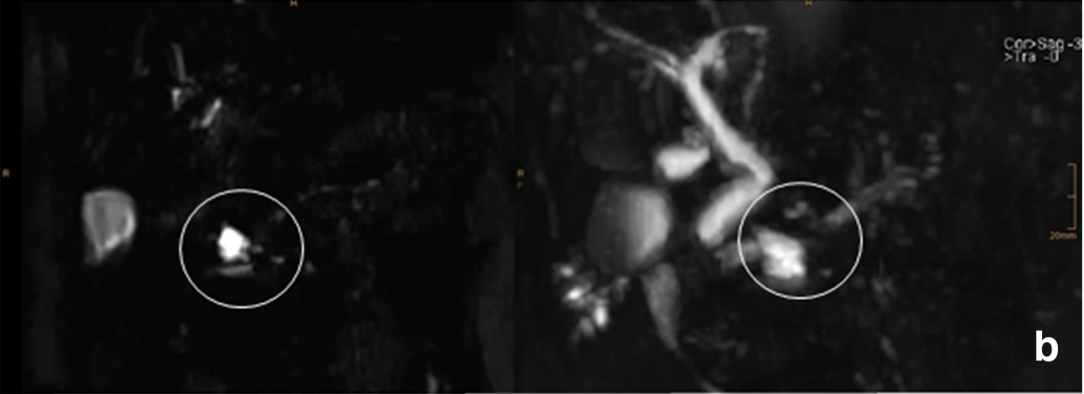

b

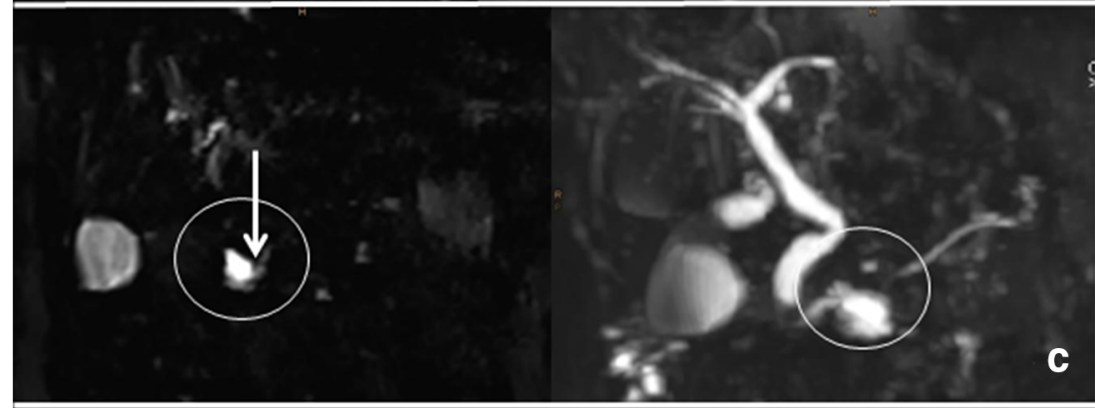

There is little literature on the evaluation of CS-MRCP sequences at $1.5 \mathrm{~T}$. The study by Taron et al evaluated CSMRCP sequences with $1.5 \mathrm{~T}$ and $3 \mathrm{~T}$ [20]. In addition to a phantom study, they evaluated 66 patients (19 for IPMN) with a conventional 3D NT-SPACE and a prototype 3D NT/BH CSSPACE. The BH CS-SPACE resulted in significantly inferior

Table 4 Inter-rater agreement

\begin{tabular}{|c|c|c|c|c|c|c|}
\hline Sequence & Image quality & Duct sharpness & Duct visualization & Lesion conspicuity & Confidence & Communication \\
\hline NT-SPACE & $0.874(0.86-0.89$ & $0.914(0.90-0.93)$ & $0.855(0.84-0.87)$ & $0.897(0.89-0.91)$ & $0.78(0.76-0.80)$ & $0.759(0.74-0.78)$ \\
\hline BHL-CS-SPACE & $0.896(0.88-0.91)$ & $0.869(0.85-0.89)$ & $0.856(0.84-0.87)$ & $0.892(0.88-0.91)$ & $0.756(0.73-0.78)$ & $0.84(0.82-0.86)$ \\
\hline BHS-CS-SPACE & $0.885(0.87-0.89)$ & $0.915(0.90-0.93)$ & $0.886(0.87-0.90)$ & $0.851(0.84-0.86)$ & $0.746(0.72-0.77)$ & $0.862(0.85-0.88)$ \\
\hline NT-CS-SPACE & $0.86(0.85-0.87)$ & $0.877(0.86-0.89)$ & $0.826(0.81-0.84)$ & $0.832(0.82-0.85)$ & $0.715(0.69-0.74)$ & $0.837(0.82-0.86)$ \\
\hline
\end{tabular}

$p$ value was $<0.05$ for all evaluations; numbers in parentheses are $95 \%$ confidence interval 
ratings in all aspects compared with NT-CS-SPACE, and the NTCS-SPACE was found superior to the standard NT-SPACE. These results are in contradiction to ours. We had no comparison to 3-T images, but, in our patient cohort, the CS BH sequences were superior to CS NT sequences. Further CS NT sequences had the most inferior ratings of all tested sequences. One reason for the different results could possibly be seen in the different approach of rating the sequences. In our study, the main focus was on the representation of the pancreatic duct, and we did not evaluate, e.g., the visibility of peripheral structures in the biliary system. Another reason for our good results with the $\mathrm{CS}$ BH sequence might also be our relatively old patient population (mean age 68 years; range, 46-89 years). Especially for these patients, the very low breath-hold time of only $7 \mathrm{~s}$ might be beneficial. It has been shown that short examination time in general leads to better image quality [21]. It has to be noted that in agreement with our results, other studies as well found better results with the BH CS sequences at $3 \mathrm{~T}$, compared with NT CS sequences [22, 23].

Tokoro et al found that the BH CS-MRCP sequence brings added value to standard NT MRCP, because it compensates the image deterioration of NT MRCP [24]. In detail, they found 43/ 113 cases with clinically inadequate image quality with the NT MRCP. The image quality in $13 / 43$ cases could be improved to clinically adequate by adding the BH CS-MRCP sequence.

Image reconstruction for the CS sequences is usually very long (approx. $5 \mathrm{~min}$ ) [17]. By integrating the CS sequences as the last sequence into our standard protocol, the time of patient change was used for reconstruction without delaying the routine workflow. With this approach, however, there is no possibility to check image quality and to eventually rerun the sequence, as the images were only available when the next patient was already in the examination room. The time of image reconstruction was not further evaluated in our study, because our MR scanner was equipped with the standard hardware which was not optimized to reconstruct demanding compressed sensing applications.

There are limitations to this study. First, this is a retrospective study. Second, we did not perform correlation with concrete pathology; e.g., the diagnosis of BD-IPMN was not further assessed nor was there any correlation with other diagnostic procedures. Our study aimed at finding an ideal sequence and comparing the performance between several sequences. Third, we had no records of patient cooperation. This might be of interest as we hypothesized that the age could be a relevant factor for image quality which was not proven or correlated with the general condition of our patients. Finally, our study population was rather small.

\section{Conclusion}

MRCP imaging with CS-SPACE for the evaluation of BDIPMN at $1.5 \mathrm{~T}$ provides the best results using a short breath- hold sequence with results comparable with conventional NTSPACE and superior to NT-CS-SPACE. This approach is feasible and an excellent alternative to standard 3D NTMRCP sequences, especially concerning the diagnosis and follow-up of BD-IPMN.

Funding information Open access funding provided by University of Innsbruck and Medical University of Innsbruck. The authors state that this work has not received any funding.

\section{Compliance with ethical standards}

Guarantor The scientific guarantor of this publication is PD Dr. Benjamin Henniner.

Conflict of interest The authors of this manuscript declare relationships with the following companies: Siemens Healthineers.

Statistics and biometry One of the authors has significant statistical expertise.

Informed consent Written informed consent was not required for this study because of its retrospective character and further written informed consent was waived by the institutional review board.

Ethical approval Institutional review board approval was not required because of the retrospective character.

\section{Methodology \\ -retrospective \\ -observational \\ -performed at one institution}

Open Access This article is licensed under a Creative Commons Attribution 4.0 International License, which permits use, sharing, adaptation, distribution and reproduction in any medium or format, as long as you give appropriate credit to the original author(s) and the source, provide a link to the Creative Commons licence, and indicate if changes were made. The images or other third party material in this article are included in the article's Creative Commons licence, unless indicated otherwise in a credit line to the material. If material is not included in the article's Creative Commons licence and your intended use is not permitted by statutory regulation or exceeds the permitted use, you will need to obtain permission directly from the copyright holder. To view a copy of this licence, visit http://creativecommons.org/licenses/by/4.0/.

\section{References}

1. O’Neill E, Hammond N, Miller FH (2014) MR imaging of the pancreas. Radiol Clin North Am 52:757-777

2. Tanaka M, Fernandez-Del Castillo C, Kamisawa T et al (2017) Revisions of international consensus Fukuoka guidelines for the management of IPMN of the pancreas. Pancreatology 17:738-753

3. Anupindi SA, Victoria T (2008) Magnetic resonance cholangiopancreatography: techniques and applications. Magn Reson Imaging Clin N Am 16:453-466 v

4. Kim SH, Lee JM, Lee ES et al (2015) Intraductal papillary mucinous neoplasms of the pancreas: evaluation of malignant potential 
and surgical resectability by using MR imaging with MR cholangiography. Radiology 274:723-733

5. Sodickson A, Mortele KJ, Barish MA, Zou KH, Thibodeau S, Tempany CM (2006) Three-dimensional fast-recovery fast spinecho MRCP: comparison with two-dimensional single-shot fast spin-echo techniques. Radiology 238:549-559

6. Liu K, Xie P, Peng W, Zhou Z (2015) Magnetic resonance cholangiopancreatography: comparison of two- and threedimensional sequences for the assessment of pancreatic cystic lesions. Oncol Lett 9:1917-1921

7. Megibow AJ, Baker ME, Morgan DE et al (2017) Management of incidental pancreatic cysts: a white paper of the ACR Incidental Findings Committee. J Am Coll Radiol 14:911-923

8. Arizono S, Isoda H, Maetani YS et al (2008) High-spatialresolution three-dimensional MR cholangiography using a highsampling-efficiency technique (SPACE) at $3 \mathrm{~T}$ : comparison with the conventional constant flip angle sequence in healthy volunteers. J Magn Reson Imaging 28:685-690

9. Lustig M, Donoho D, Pauly JM (2007) Sparse MRI: the application of compressed sensing for rapid MR imaging. Magn Reson Med 58:1182-1195

10. Henninger B, Raithel E, Kranewitter C, Steurer M, Jaschke W, Kremser C (2018) Evaluation of an accelerated 3D SPACE sequence with compressed sensing and free-stop scan mode for imaging of the knee. Eur J Radiol 102:74-82

11. Vincenti G, Monney P, Chaptinel J et al (2014) Compressed sensing single-breath-hold CMR for fast quantification of LV function, volumes, and mass. JACC Cardiovasc Imaging 7:882-892

12. Chandarana H, Doshi AM, Shanbhogue A et al (2016) Threedimensional MR cholangiopancreatography in a breath hold with sparsity-based reconstruction of highly undersampled data. Radiology 280:585-594

13. Seo N, Park MS, Han K et al (2017) Feasibility of 3D navigatortriggered magnetic resonance cholangiopancreatography with combined parallel imaging and compressed sensing reconstruction at 3 T. J Magn Reson Imaging 46:1289-1297

14. Nagata S, Goshima S, Noda Y et al (2019) Magnetic resonance cholangiopancreatography using optimized integrated combination with parallel imaging and compressed sensing technique. Abdom Radiol (NY) 44:1766-1772

15. (2006) R Development Core Team (2006), Vienna, Austria, URL: http://www.R-project.org, Version 3.5.1.
16. Matthias Gamer JL, and Ian Fellows Puspendra Singh (2012). irr: Various coefficients of interrater reliability and agreement. R package version $084 \mathrm{https}: / /$ www.CRANR-projectorg/package=irr

17. Yoon LS, Catalano OA, Fritz S, Ferrone CR, Hahn PF, Sahani DV (2009) Another dimension in magnetic resonance cholangiopancreatography: comparison of 2- and 3-dimensional magnetic resonance cholangiopancreatography for the evaluation of intraductal papillary mucinous neoplasm of the pancreas. $J$ Comput Assist Tomogr 33:363-368

18. Kwon H, Reid S, Kim D, Lee S, Cho J, Oh J (2018) Diagnosing common bile duct obstruction: comparison of image quality and diagnostic performance of three-dimensional magnetic resonance cholangiopancreatography with and without compressed sensing. Abdom Radiol (NY) 43:2255-2261

19. Furlan A, Bayram E, Thangasamy S, Barley D, Dasyam A (2018) Application of compressed sensing to $3 \mathrm{D}$ magnetic resonance cholangiopancreatography for the evaluation of pancreatic cystic lesions. Magn Reson Imaging 52:131-136

20. Taron J, Weiss J, Notohamiprodjo M et al (2018) Acceleration of magnetic resonance cholangiopancreatography using compressed sensing at 1.5 and $3 \mathrm{~T}$ : a clinical feasibility study. Invest Radiol 53:681-688

21. Zhu L, Wu X, Sun Z et al (2018) Compressed-sensing accelerated 3-dimensional magnetic resonance cholangiopancreatography: application in suspected pancreatic diseases. Invest Radiol 53:150 157

22. Yoon JH, Lee SM, Kang HJ et al (2017) Clinical feasibility of 3dimensional magnetic resonance cholangiopancreatography using compressed sensing: comparison of image quality and diagnostic performance. Invest Radiol 52:612-619

23. Zhu L, Xue H, Sun Z et al (2018) Modified breath-hold compressed-sensing 3D MR cholangiopancreatography with a small field-of-view and high resolution acquisition: clinical feasibility in biliary and pancreatic disorders. J Magn Reson Imaging 48:13891399

24. Tokoro H, Yamada A, Suzuki T et al (2020) Usefulness of breathhold compressed sensing accelerated three-dimensional magnetic resonance cholangiopancreatography (MRCP) added to respiratory-gating conventional MRCP. Eur J Radiol 122:108765

Publisher's note Springer Nature remains neutral with regard to jurisdictional claims in published maps and institutional affiliations. 